\title{
Effect of Nebulizer Designs on Aerosol Delivery During Non-Invasive Mechanical Ventilation: A Modeling Study of In Vitro Data
}

\author{
Haitham Saeed · Ahmed A. Elberry • Abeer Salah Eldin • Hoda Rabea • \\ Mohamed E. A. Abdelrahim
}

Received: January 31, 2017 / Published online: March 23, 2017

(c) The Author(s) 2017. This article is an open access publication

\section{ABSTRACT}

Introduction: Nebulizers used to deliver aerosol during non-invasive mechanical ventilation (NIV) have many types, each type has many different designs. This study aimed to determine the effect of nebulizer deign on aerosol delivery in NIV.

Methods: Different designs of jet nebulizer (JN) and vibrating mesh nebulizer (VMN) were tested by placing $1 \mathrm{ml}$ of salbutamol in a nebulizer chamber operated in an in vitro NIV setting using a breathing simulator with adult settings (inhalation-exhalation ratio $1: 3,15$ breaths/

Enhanced content To view enhanced content for this article go to http://www.medengine.com/Redeem/ 34F7F0601749963B.

H. Saeed · H. Rabea · M. E. A. Abdelrahim Clinical Pharmacy Department, Faculty of Pharmacy, Beni-suef University, Beni-suef, Egypt

\section{A. A. Elberry}

Clinical Pharmacology Department, Faculty of Medicine, Beni-suef University, Beni-suef, Egypt

A. S. Eldin

Respiratory Department, Faculty of Medicine, Beni-suef University, Beni-suef, Egypt

M. E. A. Abdelrahim ( $\square)$

Clinical Pharmacy Department, Faculty of

Pharmacy, Ahram Canadian University, Giza, Egypt

e-mail: mohamedemam9@yahoo.com min and tidal volume $500 \mathrm{ml}$ ) and mechanical ventilator (MV) set at spontaneous mode with a peak inspiratory pressure of $20 \mathrm{~cm} \mathrm{H}_{2} \mathrm{O}$ and a peak expiratory pressure of $5 \mathrm{~cm} \mathrm{H}_{2} \mathrm{O}$. Inhalation filters were placed before the breathing simulator to collect emitted aerosol (inhalable dose). Also, amounts of drug left in nebulizer chamber (residual dose) were collected and analyzed for salbutamol content. Statistical data analysis and modeling was performed on the results.

Results: The vibrating mesh nebulizer delivered significantly higher inhalable doses and lower residual dose compared to JN $(p<0.001)$. Inhalable doses of salbutamol and residual dose differed significantly $(p<0.05)$ for different designs of JN. Delivery via VMN was not affected by the difference in designs. Data modeling showed no change by changing VMN type but changing JN type resulted in a sharp change in the inhaled and residual doses.

Conclusions: Nebulizer design has a great role in efficacy of JN but not VMN and different types of JN should not considered interchanges without dose adjustment. Modeling can optimize these results for better aerosol delivery.

Keywords: Fill volume; Modeling; Nebulizer; Neural networks; Non-invasive mechanical ventilation (NIV) 


\section{INTRODUCTION}

There are three main types of nebulizers (jet, ultrasonic, and vibrating mesh nebulizer). The jet nebulizer (JN) is the most commonly used type [1] due to its low cost, but the main disadvantage of $\mathrm{JN}$ is its very low percent of emitted drug. Less than $10 \%$ of total dose could be delivered to the patient and in some situations it could be 5\% [2]. This low percent of delivered dose may affect therapy outcomes of the patient [3]. This obstacle may be overcome with a vibrating mesh nebulizer (VMN) [4, 5], which is expensive, but more efficient in delivery than the JN [6]. JN and VMN have various brands with different designs. The jet is operated by air pressure [6]. Nozzle cover and nebulizer chamber shape or sizes are the main parts that differ from one jet nebulizer to another [6]. For vibrating mesh nebulizers, the shape of solution container (the molding of the nebulizer chamber) is the main difference in design, with the same vibrating mesh technology.

Delivery of aerosol to mechanically ventilated patients using JN and VMN is a widespread practice. Aerosol delivery in non-invasive ventilation (NIV) was proven to be of benefit and has been well studied [5, 7-11]. However, the effect of the change of design of nebulizers with the same mechanism on delivery has not been studied much. The aim of this study was therefore to evaluate the effect of different nebulizer designs on aerosol delivery to non-invasive ventilated patients.

\section{METHODS}

Three differently brand, with different design, of JN and another three differently brand, with different design, of VMN were used in the study. The JN used were JN A (Hospital and Home Care, China), JN B (Dolphin Medical, Turkey) and JN C (Philips Respironics, UK) operated with an air flow of $6 \mathrm{l} / \mathrm{min}$ into the nebulizer to aerosolize the respirable solution. The VMNs used were Aerogen Pro [PRO] Nebulizer and Aerogen Solo [SOLO] Nebulizer (Aerogen Limited, Galway, Ireland), and NIVO Nebulizer (Aerogen/Philips, MA, USA). Figure 1 represents a schematic diagram of the six nebulizers that were studied.

Each nebulizer with its T-piece was connected to the NIV in vitro model as shown in Fig. 2 . The NIV in vitro model consisted of a breathing simulator (5600i, Michigan Instruments, USA) with adult settings (inhalation: exhalation ratio 1:3, 15 breaths/min and tidal volume $500 \mathrm{ml}$ ), ventilation circuit, consisted of a smooth 22-mm internal diameter tubing of $180 \mathrm{~cm}$ length, and ventilator (Nippy2, B and D Electromedical, Warwickshire, UK). The ventilator was set in spontaneous mode with a peak inspiratory pressure of $20 \mathrm{~cm} \mathrm{H}_{2} \mathrm{O}$ and a peak expiratory pressure of $5 \mathrm{~cm} \mathrm{H}_{2} \mathrm{O}$. A filter was placed in a filter holder (PariGmbH, Starnberg, Germany) fixed before the breathing simulator for collection of inhalable dose from each nebulizer studied that could be delivered to the breathing simulator representing the patient.

For each nebulizer, ten runs were performed. During each run, $1 \mathrm{ml}$ of salbutamol (Farcolin respirator solution, $5000 \mu \mathrm{g} / \mathrm{ml}$; Pharco Pharmaceuticals, Egypt) was placed inside the nebulizer chamber. We chose this small fixed volume to determine the real effect of the nebulizer design on aerosol delivery since the residual dose left in the nebulization chamber differs from one nebulizer to another [12]. The breathing simulator and ventilator were turned on $30 \mathrm{~s}$ before operating the nebulizers. For JNs, they were operated until sputter but VMNs were operated until no vapors were seen in the T-piece.

Filters were collected and rinsed in 90\% acetonitrile $(\mathrm{v} / \mathrm{v})$ and then sonicated and the nebulizer chamber was rinsed with a constant volume of $90 \%$ acetonitrile (v/v). High-performance liquid chromatography (HPLC) with UV detection was used to determine the amounts of salbutamol obtained from filters left in the nebulizer chamber. The method used an Agilent 1260 infinity diode array detector VL (G131SD, Agilent, USA) set at $225 \mathrm{~nm}$ to quantify amounts of salbutamol. A mobile phase consisted of $(90: 10 \mathrm{v} / \mathrm{v})$ acetonitrile and water mixture [containing $0.1 \%$ phosphoric acid] was pumped at flow rate $1 \mathrm{ml} / \mathrm{min}$ by Agilent 1260 infinity preparative pump (G1361A, Agilent, USA), through c18 column (a $25 \times 4.6 \mathrm{~mm}$ ZORBAX Eclipse Plus, Agilent, USA) with $100-\mu \mathrm{l}$ 

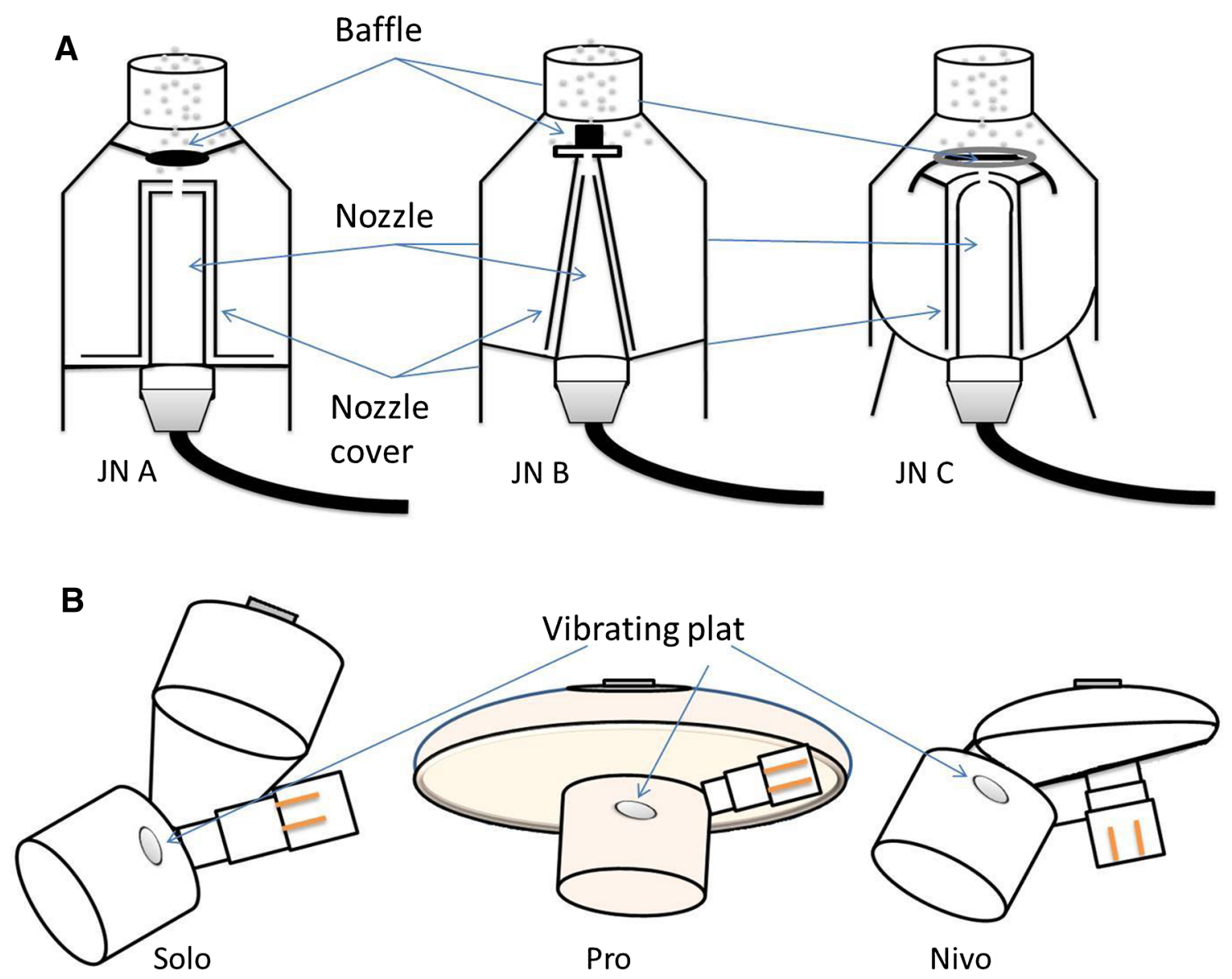

Fig. 1 Schematic design of the six different nebulizers studied

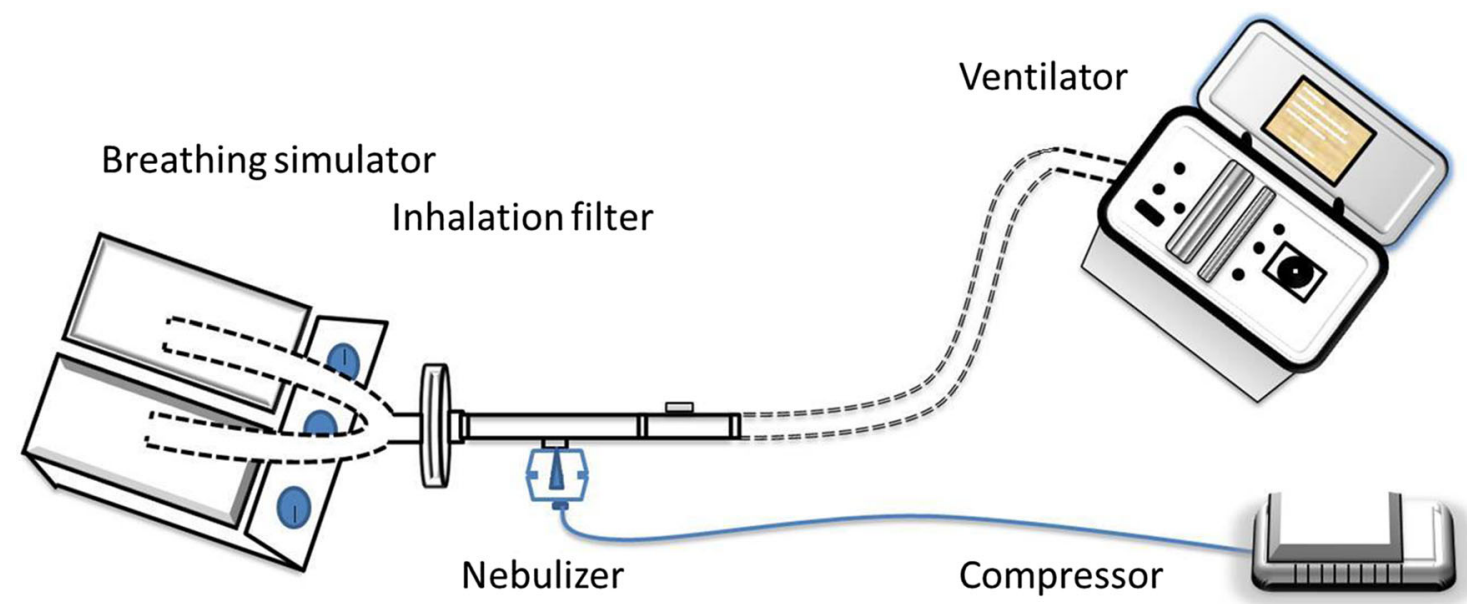

Fig. 2 Schematic design of in vitro model setting

injection volume. The lower limit of detection was $0.35 \mu \mathrm{g} / \mathrm{ml}$ and the lower limit of quantification was $2.55 \mu \mathrm{g} / \mathrm{ml}$.
Statistical analysis of data was done using two-way analysis of variance (ANOVA) with IBM SPSS V.22 (SPSS Inc., Chicago, IL, USA). 
Data modeling using Design Expert 7.0.0 (Stat-Ease Inc., Minneapolis, MN, USA) with model inputs (nebulizer type and nebulizer design) and outputs (inhalation and nebulizer chamber amounts of salbutamol). Input data were encoded numerically, for nebulizers, JN (1) and VMN (2); JN designs, JN A (0.1), JN B (0.2), and JN C (0.3); and VMN designs Solo (0.1), Pro (0.2), and Nivo (0.3).

The analysis in this article is based on previously conducted in vitro studies and does not involve any new studies of human or animal subjects performed by any of the authors.

\section{RESULTS}

Table 1 and Fig. 3 show the amount of salbutamol recovered from each inhalation filter (inhalable dose) and nebulizer chamber (residual dose) of the six nebulizers expressed as mean (SD) $\mu \mathrm{g}$.

The three VMNs studied had a significantly higher $(p<0.001)$ amount of salbutamol deposited on the inhalation filter (inhaled dose) and a significantly lower amount of salbutamol remaining in the nebulizer chamber (residual dose) than the three studied JN $(p<0.001)$.

Designs of jet nebulizers different design had a significant effect on inhaled dose and residual dose $(p<0.05)$. JN C had the highest emitted dose while JN A had the lowest one $(p<0.05)$. However, there were no significant differences between the three VMNs studied, as shown in Table 1 and Fig. 3.

Modeling of the data showed a successful model for validation of current data and could help in the prediction of further data. This is indicated by low design stander error as shown in Fig. 4 and the model high predicted correlation coefficient $\left(R^{2}\right)$, as shown in Table 2 . Similar to statistical comparison, data modeling showed the significant effect of nebulizer design on JN efficacy. Response surface plots of the effect of nebulizer type and design on inhaled dose is shown in Fig. 5 and on residual dose in Fig. 6.

Figures 5 and 6 show that no change was observed by changing the type of the VMN but changing the JN type resulted in a sharp change 
Fig. 3 Mean (SD) inhalable dose and residual dose in $\mu \mathrm{g}$ from the six different nebulizers studied

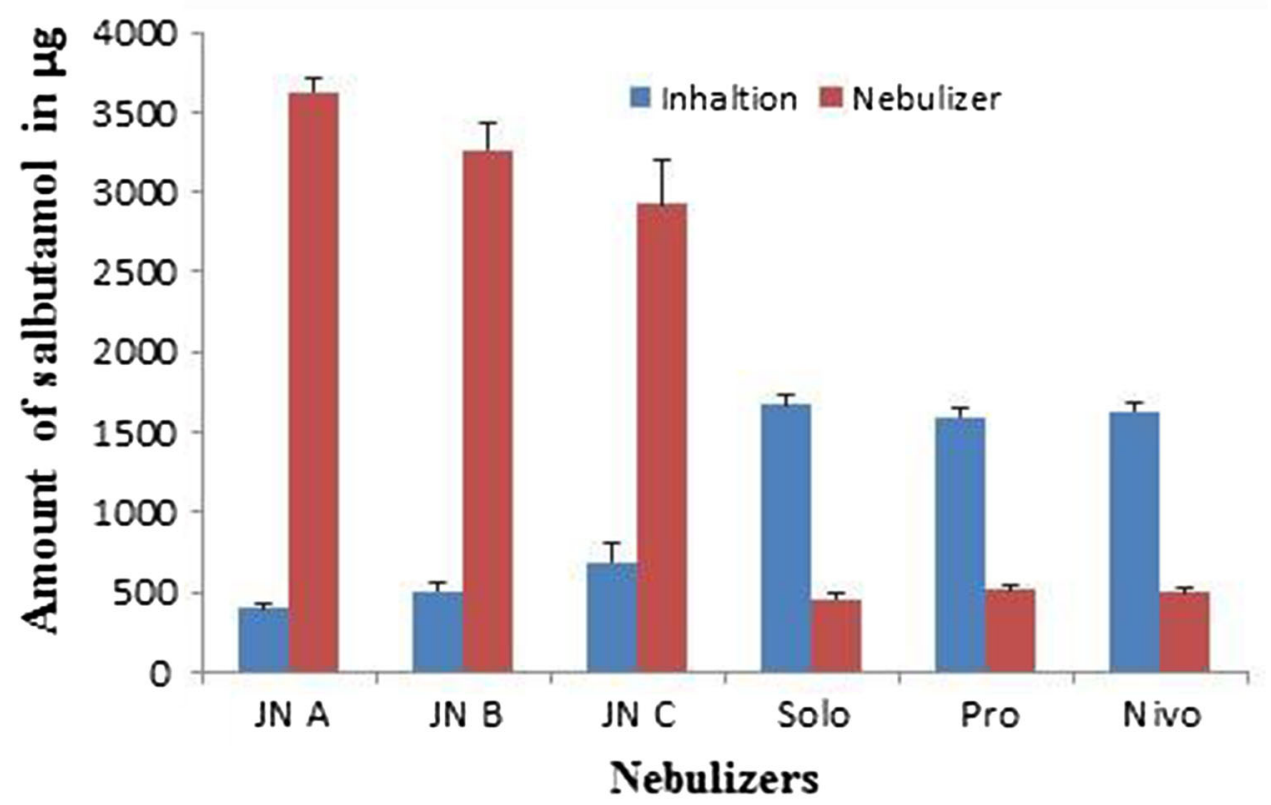

Fig. 4 Model stander error for nebulizers and designs

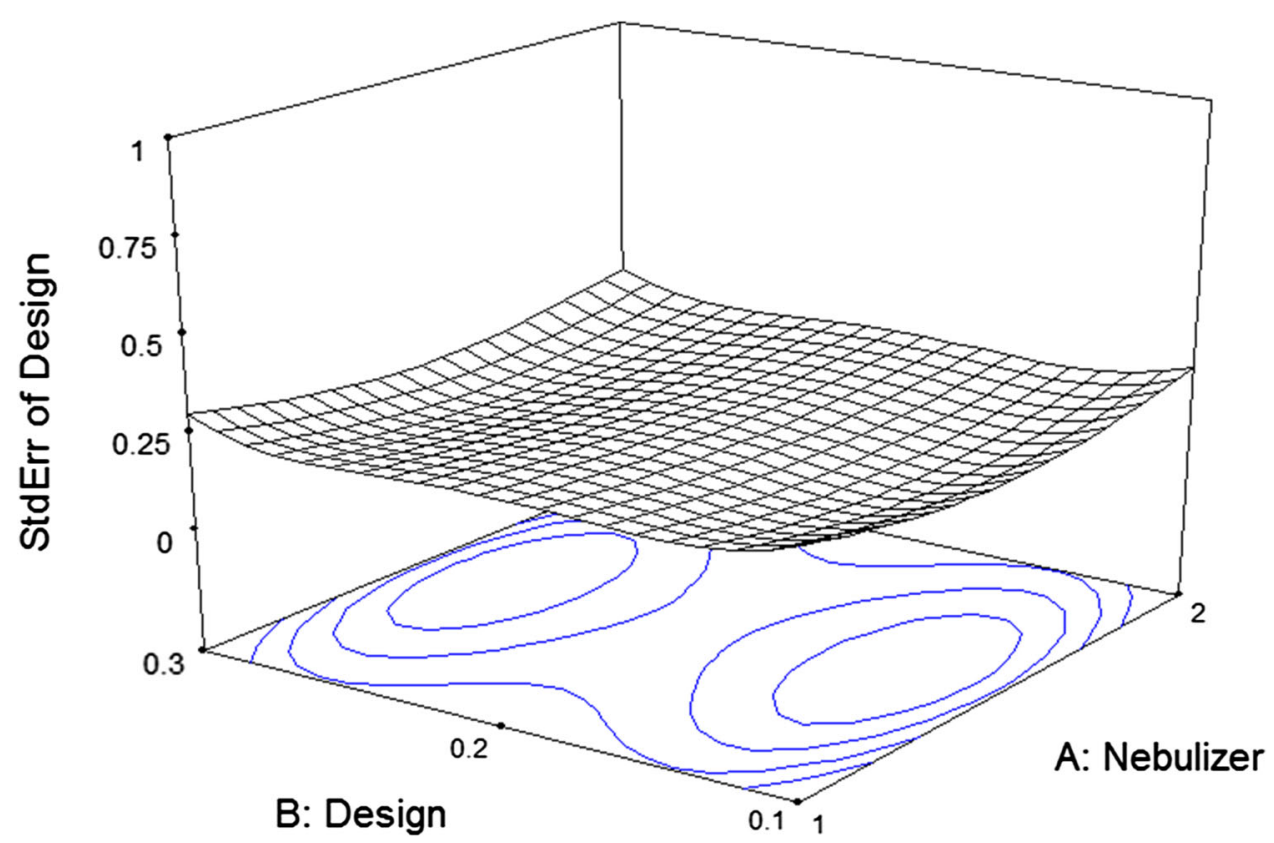

in the inhaled dose and residual dose. Figures 5 and 6 also show that the VMN had a much better inhaled dose and lower residual dose than JN. The emitted dose from JN decreased from the highest value for JN C to the lowest of $\mathrm{JN}$ A, showing the role of nebulizer design on inhalable dose, while the upper border of Fig. 5 did not show any obvious decline, indicating that efficacy of VMN was not affected by the nebulizer designs. Also, the upper border of Fig. 6 represents the amounts of drug that remained in the JN chambers, which were higher than those of VMNs, which are shown at the lower border of Fig. 6 . 
Table 2 Model ANOVA statistics for inhalation and nebulizer chamber model

\begin{tabular}{llll}
\hline Source & $\boldsymbol{F}$ value & $\begin{array}{l}\boldsymbol{p} \text { value } \\
\text { Probability }>\boldsymbol{F}\end{array}$ \\
\hline Inhalation dose & 132.29 & $<0.0001$ \\
Model & 387.73 & $<0.0001$ & 0.8763 \\
Nebulizer & 3.15 & 0.0812 & 0.8697 \\
Design & 216.71 & $R$-squared & 0.8558 \\
Standard deviation & 1080.17 & Adjusted $R$ squared & \\
Mean & 20.06 & Predicted $R$ squared & \\
CV\% & 22.689 & & \\
Adequate precision & & $<0.0001$ & \\
Nebulizer chamber & 210.87 & $<0.0001$ & 0.9187 \\
Model & 619.54 & 0.0201 & 0.9143 \\
Nebulizer & 5.73 & $R$ squared \\
Design & 432.90 & Adjusted $R$ squared & 0.9055 \\
Standard deviation & 1882.18 & Predicted $R$ squared & \\
Mean & 23.00 & & \\
CV\% & 28.212 & & \\
Adequate precision & & & \\
\hline
\end{tabular}

$C V$ coefficient of variation

Fig. 5 Response surface plot of the relation between inhalable doses and different nebulizer designs of JN and VMN

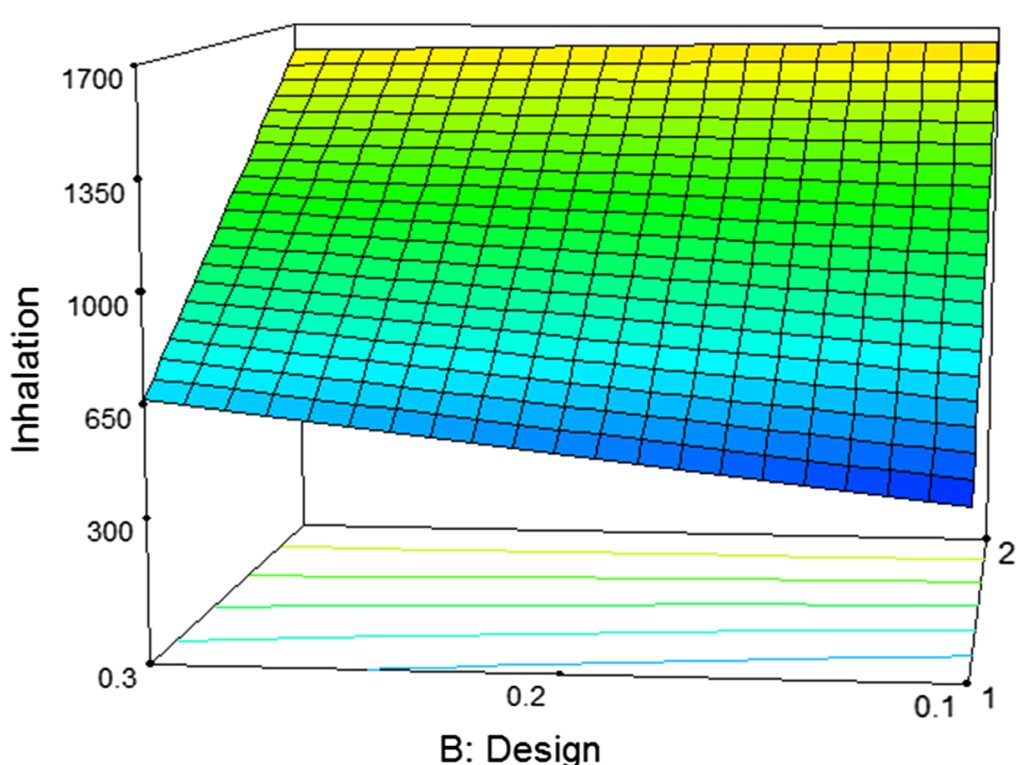

A: Nebulizer

B: Design 
Fig. 6 Response surface plot of the relation between residual dose and different nebulizer designs of JN and VMN

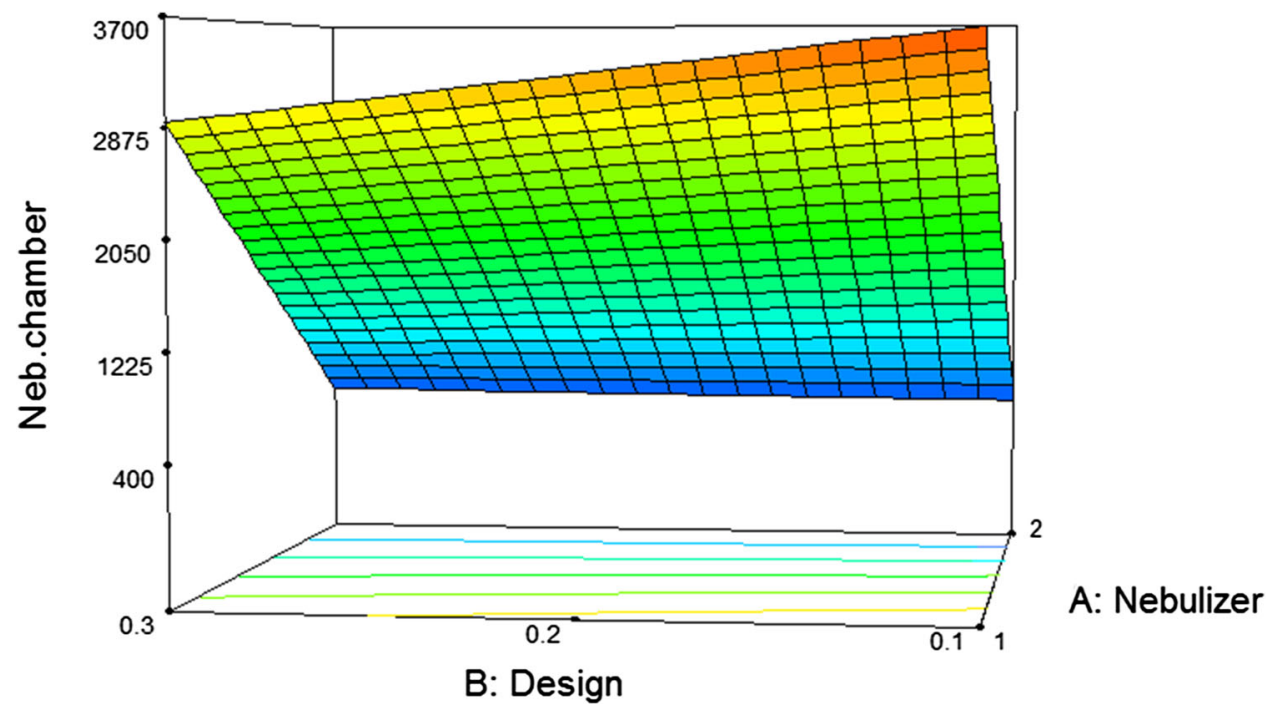

\section{DISCUSSION}

VMNs again showed much better efficiency in aerosol delivery compared to $\mathrm{JN}$, resulting in higher inhaled dose $(p<0.001)$ and lower residual dose $(p<0.001)$ [13-15].

Significantly difference inhaled dose from different $\mathrm{JN}$ designs $(p<0.05)$ demonstrate the effect of JN design on efficacy of nebulizer, which could affect therapeutic outcomes. JN C had the highest inhaled dose compared to JN A and JN B $(p<0.05)$ and JN B was more efficient than JN A $(p<0.05)$. It was noted for JN A, which has the lowest emitted dose, that the flat end of the nozzle cover had a role in low efficacy because large drops of the drug were settled on the upper face, preventing them from entering the area between the nozzle and its cover [6]. Therefore, different JN designs have a great role in nebulizer efficacy, as previously shown in a study carried out on the available brands of JN in France. They showed that different nebulizer brands had different efficacy on delivering aerosol to cystic fibrosis patients [12]. Their findings support our results as well as the other study, which showed that even different batches of the same JN resulted in different inhaled doses [16], especially with small fill volume, which is considered a very important factor affecting aerosol delivery due to residual dose remaining in the nebulizer chamber at the end of nebulization $[4,5,17]$. This is why we used a fixed low volume of salbutamol ( $1 \mathrm{ml})$ for all the nebulizers to evaluate efficacy of each device on deliver optimum amounts of drug with low volumes. Therefore, we recommend increasing the fill volume of the $\mathrm{JN}$ to overcome this difference.

However, there was no significant effect of nebulizer change on inhaled dose from VMNs which have a different operating mechanism from JN. JN aerosolizes by the effect of air pressure that passes through a nozzle fixed in the nebulizer chamber. The velocity of the passing gas causes the pressure to drop in the area between the nozzle and the nozzle cover, which is called the "Venturi effect" [6]. This drop in pressure leads the respirable solution to path through this area around the nozzle upward by Bernoulli effect forming a thin layer of solution that converts to fine particle then is emitted from the nebulizer chamber toward the patient [6]. Large droplets will be settled on the nebulizer chamber wall or nozzle cover to be returned to the reservoir solution.

However, the vibrating mesh plat consists of a piezoelectric element fixed at the bottom of the nebulizer chamber that vibrates in response to electric current [6, 18, 19]. Upward and downward movement of vibrating plat convert respirable solution in nebulizer chamber to fine droplets emitted out toward patient [20]. According to this mechanism of operation, 
which is the same for all VMNs brands, variability between different VMNs designs could be of less significance on aerosol delivery compared to JN, which is affected by the shape or size of the nozzle and also the shape of the nebulizer chamber. So the interchange of the VMNs, but not JNs, is possible with no fear of variation of the delivered dose [21].

When looking at the modeling results, the models' $F$ values of inhaled dose and residual dose were 132.29 and 210.87 , respectively. This implies that these models are significant and could be used successfully for validation and prediction of optimization of aerosol delivery, as there is only a $0.01 \%$ chance that a "model $F$ value" could occur due to noise. Also, a low standard error of model, shown from Fig. 4, reflects a non-significant lack of fit. Values of "Probability $>F$ " less than 0.05 indicate the model terms are significant. So, nebulizers, and nebulizers designs interation are significant model terms. The "predicted $R$ squared" of 0.8558 and 0.9055 are in reasonable agreement with the "adjusted $R$ squared" of 0.8697 and 0.9143 for inhalation and nebulizer chamber, respectively [21, 22]. "Adequate precision" measures the signal-to-noise ratio. Ratios of 22.689 and 28.212 indicate an adequate signal.

Modeling response surface plots (Figs. 5 and 6) support our finding with a very sharp change in the inhaled dose and residual dose by changing the design of the JN. The sharp change observed with JNs was not observed with changing the VMN type [21], however, changing from JN to VMN resulted in much better aerosol delivery. Therefore, residual dose as well as inhalable dose emitted from nebulizers studied indicated the presence of a significant effect of designs in JN only.

\section{CONCLUSIONS}

Nebulizer design significantly affected aerosol delivery by jet nebulizers with no significant effect when using vibrating mesh nebulizers of different designs. These findings were supported by statistical comparison and modeling, which proved to be a good tool for optimizing aerosol delivery from nebulizers in NIV.

\section{ACKNOWLEDGMENTS}

No funding or sponsorship was received for this study or publication of this article. All named authors meet the International Committee of Medical Journal Editors (ICMJE) criteria for authorship for this manuscript, take responsibility for the integrity of the work as a whole, and have given final approval for the version to be published.

Disclosures. Haitham Saeed, Ahmed A. Elberry, Abeer Salah Eldin, Hoda Rabea, and Mohamed E. A. Abdelrahim declare that they have no conflicts of interest to disclose.

Authors Contributions. Haitham Saeed: Experiment, data entry, writing. Ahmed A. Alberry: Concept, study design. Abeer Salah Eldin: Concept, study design. Hoda Rabea: Concept, study design. Mohamed E. Abdelrahim: Concept, planning of study design, statistics, and writing.

Compliance with Ethics Guidelines. The analysis in this article is based on previously conducted in vitro studies and does not involve any new studies of human or animal subjects performed by any of the authors.

Data Availability. The datasets generated during and/or analyzed during the current study are available from the corresponding author on reasonable request.

Open Access. This article is distributed under the terms of the Creative Commons AttributionNonCommercial 4.0 International License (http://creativecommons.org/licenses/by-nc/4. $0 /$ ), which permits any noncommercial use, distribution, and reproduction in any medium, provided you give appropriate credit to the original author(s) and the source, provide a link 
to the Creative Commons license, and indicate if changes were made.

\section{REFERENCES}

1. Rau JL, Hess D. A guide to aerosol delivery devices for respiratory therapists. AARC: Times; 2009. p. 53.

2. Abdelrahim ME, Plant $P$, Chrystyn $H$. In vitro characterisation of the nebulised dose during non-invasive ventilation. J Pharm Pharmacol. 2010;62(8):966-72.

3. Silkstone $\mathrm{V}$, et al. An investigation of in vitro/ in vivo correlations for salbutamol nebulized by eight systems. J Aerosol Med. 2002;15(3):251-9.

4. Dhand R. Aerosol delivery during mechanical ventilation: from basic techniques to new devices. J Aerosol Med Pulm Drug Deliv. 2008;21(1):45-60.

5. Ari A, et al. Influence of nebulizer type, position, and bias flow on aerosol drug delivery in simulated pediatric and adult lung models during mechanical ventilation. Respir Care. 2010;55(7):845-51.

6. Carvalho TC, McConville JT. The function and performance of aqueous aerosol devices for inhalation therapy. J Pharm Pharmacol. 2016;68(5):556-78.

7. Berlinski A, Willis JR. Effect of tidal volume and nebulizer type and position on albuterol delivery in a pediatric model of mechanical ventilation. Respir Care. 2015;60(10):1424-30.

8. Ari A, et al. Quantifying aerosol delivery in simulated spontaneously breathing patients with tracheostomy using different humidification systems with or without exhaled humidity. Respir Care. 2016;61(5):600-6.

9. Lin HL, et al. In vitro comparison of aerosol delivery using different face masks and flow rates with a high-flow humidity system. Respir Care. 2015;60(9):1215-9.

10. Restrepo RD, Walsh BK. Humidification during invasive and noninvasive mechanical ventilation: 2012. Respir Care. 2012;57(5):782-8.

11. Hassan A, et al. In vitro characterization of the aerosolized dose during non-invasive automatic continuous positive airway pressure ventilation. Pulm Ther. 2016;2(1):115-26.

12. Vecellio L, et al. Disposable versus reusable jet nebulizers for cystic fibrosis treatment with tobramycin. J Cyst Fibros. 2011;10(2):86-92.

13. Hassan $A$, et al. In vitro/in vivo comparison of inhaled salbutamol dose delivered by jet nebulizer, vibrating mesh nebulizer and metered dose inhaler with spacer during non-invasive ventilation. Exp Lung Res. 2017;4:1-10.

14. Abdelrahim M, Plant P, Chrystyn $H$. The relative lung and systemic bioavailability of terbutaline following nebulisation in non-invasively ventilated patients. Int J Pharm. 2011;420(2):313-8.

15. ElHansy MHE, et al. In vitro aerodynamic characteristics of aerosol delivered from different inhalation methods in mechanical ventilation. Pharm Dev Technol. 2016;3:1-6.

16. Hess D, et al. Medication nebulizer performance: effects of diluent volume, nebulizer flow, and nebulizer brand. CHEST J. 1996;110(2):498-505.

17. Gardenhire D, et al. A guide to aerosol delivery devices for respiratory therapists. USA: American Association for Respiratory Care TX; 2013.

18. Elhissi A, et al. Formulations generated from ethanol-based proliposomes for delivery via medical nebulizers. J Pharm Pharmacol. 2006;58(7):887-94.

19. Lass JS, Sant A, Knoch M. New advances in aerosolised drug delivery: vibrating membrane nebuliser technology. Expert Opin Drug Deliv. 2006;3(5):693-702.

20. Watts AB, McConville JT, Williams RO III. Current therapies and technological advances in aqueous aerosol drug delivery. Drug Dev Ind Pharm. 2008;34(9):913-22.

21. Rabea $\mathrm{H}$, et al. Modelling of in vitro and in vivo performance of aerosol emitted from different vibrating mesh nebulisers in non-invasive ventilation circuit. Eur J Pharm Sci. 2017;97:182-91.

22. Hussein RRS, et al. In vitro/in vivo correlation and modeling of emitted dose and lung deposition of inhaled salbutamol from metered dose inhalers with different types of spacers in noninvasively ventilated patients. Pharm Dev Technol. 2015;4:1-10 (in press). 